\title{
Effect of Driving Signal and Temperature on Hysteresis of Magnetical Shape Memory Alloy-Based Actuator
}

\begin{abstract}
Miaolei Zhou ${ }^{a}$, Yewei Yu ${ }^{a}$, Chen Zhang ${ }^{a}$ And Shouchun Wang $^{b, *}$
${ }^{a}$ Department of Control Science and Engineering, Jilin University, Changchun 130022, P.R. China ${ }^{b}$ Department of Neurology, The First Bethune Hospital of Jilin University, Changchun 130022, P.R. China

With the development of micromotion actuation technology, smart material-based actuators are more promising in micro/nanopositioning field. As a typical example of smart material-based actuators, magnetical shape memory alloy actuator has been applied in nanopositioning stages due to its extremely innovative properties such as high precision nanoscale positioning and large stroke. However, the inherent hysteresis nonlinearity of the magnetical shape memory alloy material affects the performance of the magnetical shape memory alloy-based actuator in the field of high precision positioning. The hysteresis characteristics of the magnetical shape memory alloybased actuator are related to many factors. This paper is devoted to study the effect of driving signal and external temperature on the displacement of the magnetical shape memory alloy-based actuator. Firstly, the structure and principle of the magnetical shape memory alloy-based actuator are introduced. Then the displacement curves of the magnetical shape memory alloy-based actuator are tested under different input frequencies, amplitudes, and environment temperatures. Finally, the hysteresis characteristics of the magnetical shape memory alloy-based actuator under different driving signals and environment temperatures are analyzed.
\end{abstract}

DOI: 10.12693/APhysPolA.137.982

PACS/topics: magnetical shape memory alloy, actuator, hysteresis, rate-dependent

\section{Introduction}

Magnetical shape memory alloy (MSMA) is a ferromagnetic material that generates force and motion under moderate magnetic fields [1], as shown in Fig. 1a. Whilst not achieving the frequency of piezo-based materials or magnetostrictive materials, MSMA material gives much higher large stroke. The typical MSMA material made by nickel, manganese, and gallium is able to generate $12 \%$ strain under external loads [2]. Using the magnetic shape memory effect, MSMA can be used to design actuators, energy harvesters, and sensors, where the MSMA elongates based on the presence of a magnetic field, as shown in Fig. 1b. The elongation is fully reversible, either by applying a magnetic field at $90^{\circ}$ to the original field, or more effectively by using of a spring. Because of high precision nanoscale positioning and large stroke, MSMA-based actuator possesses distinct advantages over traditional actuators in nanophysics and multifunctional magnetic materials field $[3,4]$. However, the MSMA-based actuator is not widely used in practice fields at present due to the fact that hysteresis of the MSMA-based actuator has strong sensitivity to the change of the driving signal and the temperature in the workplace [5-7].

\section{The structure and working principle of the MSMA-based actuator}

Figure $2 \mathrm{a}$ and $\mathrm{b}$ shows the picture and structure of the MSMA-based actuator which is composed of the MSMA

*corresponding author; e-mail: wangsc13@163.com (a)
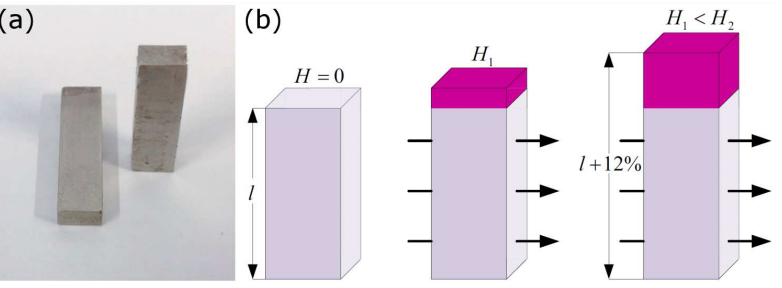

Fig. 1. (a) Picture of the MSMA material. (b) Deformation principle of the MSMA material.

material with dimensions of $2 \times 3 \times 14 \mathrm{~mm}^{3}$, a spring, coils, and a push-rod. The working principle of the MSMA-based actuator is as follows: MSMA material is placed under a movable push-rod that is connected with a spring working as returning mechanism. When the coils are supplied by the electric current, the MSMA begins to elongate under the effect of the magnetic field, thus causing the push-rod to move upward. At the same time the spring is compressed and applies a force in the opposite direction to the MSMA material. When the magnetic field begins to decrease, the MSMA will be compressed under the action of the spring and as a result, the pushrod has a downward movement.

Figure 3 shows the experimental curves of the MSMAbased actuator. Experimental data show that there is significant hysteresis nonlinearity between the input current of the actuator and the displacement of the actuator. At the initial stage, MSMA is subjected to a preload from the spring, and as the current increases the displacement of the MSMA-based actuator grows slowly (case 1). When the input current reaches a threshold, MSMA begins to elongate rapidly, and this change causes a rapid 

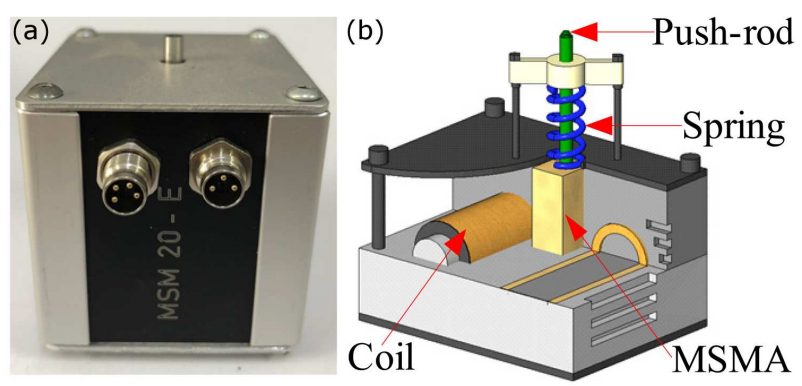

Fig. 2. (a) Picture of the MSMA-based actuator. (b) Structure of the MSMA-based actuator.

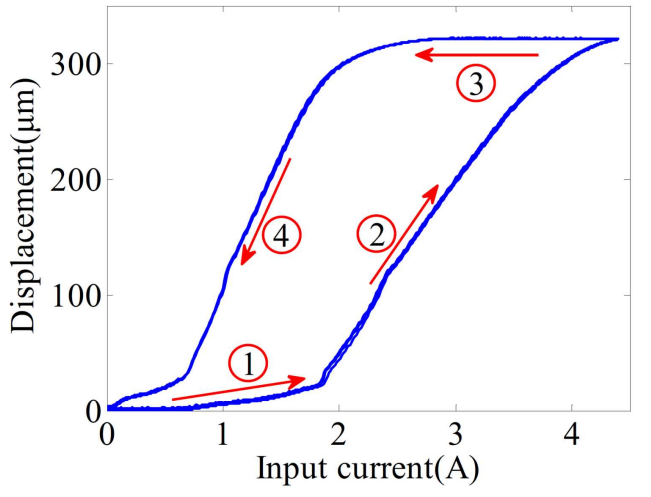

Fig. 3. Hysteresis loop of the MSMA-based actuator.

increase in the output displacement of the MSMA-based actuator (case 2). When the current begins to decrease, the length of the MSMA remains unchanged initially. Thus, the output displacement of the MSMA-based actuator remains a constant, that is, the hysteresis loop is characterized by high saturation and distinguished from other smart material based actuator (case 3). As the current continues to decrease, the displacement of the MSMA-based actuator decreases and eventually goes to zero when the current becomes to zero (case 4). Experimental details also suggest that the MSMA-based actuator has access to a giant macroscopic strain.

\section{Experimental research}

In order to test the influence of driving signal and working environment temperature on the output displacement of the MSMA-based actuator, an experimental system is built. The physical diagram of the experimental device is shown in Fig. 4. The experimental setup includes a MSMA-based actuator (built-in temperature sensor), a host computer, a linear variable differential transformer (LVDT), a data acquisition card (PCI-1716), and a programmable precision DC power.

The MSMA-based actuator is actuated by programmable precision DC power and the output displacement of the MSMA-based actuator is measured by using LVDT. To prevent overheating, a built-in temperature sensor is used to measure and monitor the real-time temperature of the MSMA material in actuator. Data

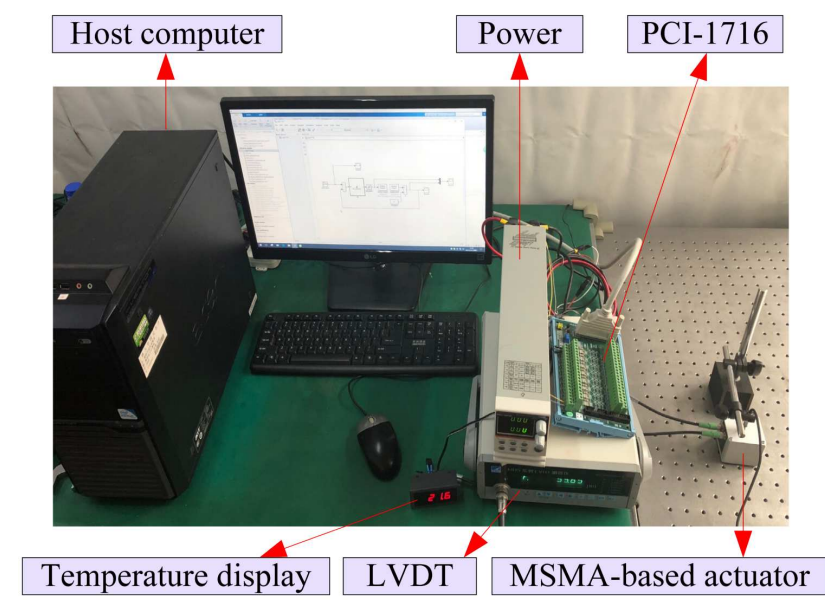

Fig. 4. Picture of experimental setup.

collection card equipped with $\mathrm{D} / \mathrm{A}$ and $\mathrm{A} / \mathrm{D}$ converters is used to collect and transmit data. The Matlab/Simulink software is used to perform different tests.

Four different tests are implemented in this section to illustrate the hysteresis characteristic of the MSMAbased actuator, and all tests are carried out at room temperature except one. Figure 5a shows the hysteresis loop of the MSMA-based actuator which is driven by variable amplitude and frequency signals. The driving signal form is

$u(t)=1.1+0.5 \sin \left(2 \pi f_{1} t+1\right)+0.6 \sin \left(2 \pi f_{2} t-\pi / 2\right)$,

with $f_{1}=1 \mathrm{~Hz}$ and $f_{2}=3 \mathrm{~Hz}$. Obviously, the hysteresis loop is wide, asymmetric, and multi-valued mapping. Moreover, the output of the MSMA-based actuator not only relates to the current input value, but also to previous input value (i.e., memory property). Figure 5b shows the case in which the MSMA-based actuator is driven by an increasing sinusoid-wave signal where the form is selected as

$$
u(t)=1.1+1.1 \sin (2 \pi f t-\pi / 2),
$$

with $f=4 \mathrm{~Hz}$. The hysteresis curve shown in Fig. 5b illustrates that saturation region of the hysteresis loop increases with increase in the driving signal amplitude.

Rate-dependent hysteresis of the MSMA-based actuator is the inherent characteristic between input current and output displacement. In Fig. 5c, as the frequency of input signal increases, the hysteresis loop becomes plump gradually and the maximum displacement of the MSMAbased actuator decreases. The trend of the change in the loop shape becomes different as the frequency grows more than about $10 \mathrm{~Hz}$. As the frequency increases further, the hysteresis loop of the MSMA-based actuator stops being plump and the area of hysteresis loop decreases.

Besides, the displacement of the MSMA-based actuator also depends on the temperature of the environment. Different temperature tests are implemented on the MSMA-based actuator, and the driving signal is selected as $u(t)=0.5 \sin (2 \pi f t-\pi / 2)+1.5$ with $f=1 \mathrm{~Hz}$. The experimental results show that different temperatures 

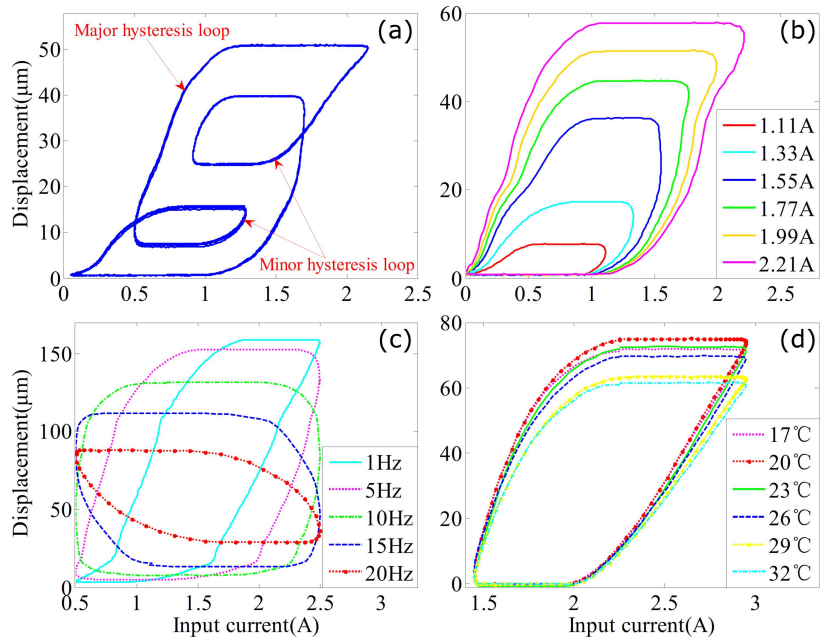

c)

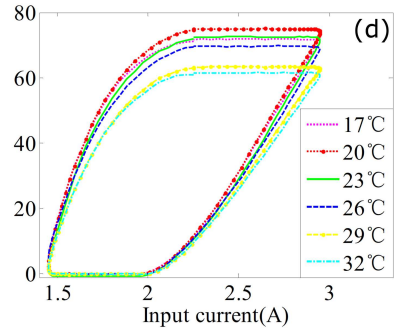

Fig. 5. Experimental curves of the MSMA-based actuator (a) driven by variable amplitude and frequency signal, (b) driven by variable amplitude signal, (c) driven by variable frequency signal, (d) at different temperature.

have a significant effect on the shape of the hysteresis curve of the MSMA-based actuator. In the temperature range of $17^{\circ} \mathrm{C}-20^{\circ} \mathrm{C}$, the maximum displacement of the MSMA-based actuator increases with increase in temperature. When the temperature is higher than $20^{\circ} \mathrm{C}$ and continues to increase, the maximum displacement of the MSMA-based actuator gradually decreases, as shown in Fig. 5d.

\section{Conclusions}

This study examines the effect of driving signal and temperature on hysteresis of the MSMA-based actuator. In the course of experiments, some effects not previously described in literature are observed. Experimental results show that the hysteresis behavior of the
MSMA-based actuator highly depend on the change of input signal. Additional, temperature is also one of the important factors affecting the displacement output of the MSMA-based actuator. The results of experiment are valuable to engage in study of hysteresis characteristic of smart materials and promote the application of the MSMA-based actuator in precise positioning field in the future.

\section{Acknowledgments}

This work was supported in part by the National Natural Science Foundation of China under Grant 51675228 and the Program of Science and Technology Development Plan of Jilin Province of China under Grants 20180101052JC, 20190303020SF.

\section{References}

[1] V.A. Chernenko, E. Cesari, V.V. Kokorin, I.N. Vitenko, Scr. Metall. Mater. 33, 1239 (2013).

[2] A. Sozinov, N. Lanska, A. Soroka, W. Zou, Appl. Phys. Lett. 102, 021902 (2013).

[3] O. Heczko, V. Bradshaw, Acta Phys. Pol. A 131, 1063 (2017).

[4] K. Schluter, B. Holz, A. Raatz, Adv. Eng. Mater. 14, $682(2012)$.

[5] S. Shevyrtalov, K. Chichay, P. Ershov, V. Khovaylo, A. Zhukov, V. Zhukova, V. Rodionova, Acta Phys. Pol. A 127, 603 (2015).

[6] L. Riccardi, D. Naso, H. Janocha, B. Turchiano, Mechatronics 22, 568 (2012).

[7] Y.W. Yu, C. Zhang, M.L. Zhou, IEEE Trans. Nanotechnol. 19, 19310051 (2020). 